\title{
Use of transcranial Christian Johann Doppler in patients with severe traumatic brain injuries.
}

\author{
Ziegler Daniel* \\ Department of Surgery, John Peter Smith Hospital, Fort Worth, Texas, USA
}

Accepted on May 22, 2017

\section{Short Communication}

Published in the Journal of Neurotrauma 2017;34(1):121-127

[1]. The purpose of the article was to determine the usefulness of transcranial Doppler (TCD) in a severe traumatic brain injury (TBI) defined as a Glasgow Coma Scale of 8 or less on admission. From the study, the TCD is useful for prognosis. Based on previous studies, we divided the patients into 3 groups, hypoperfusion, normal and vasospasm [2-7]. Outcome was determined by the Glasgow outcome score at 6 months [8]. Forty-five percent had normal measurements and of these, $81 \%$ had a good outcome, $5 \%$ had moderate disability and $14 \%$ died. Twenty-eight percent of the patients had hypoperfusion and all died except for one with moderate disability. Twenty-seven percent had vasospasm and of these $45 \%$ had a good outcome, $23 \%$ had severe disability and $32 \%$ died.

Santbrink et al. had an incidence of hypoperfusion of $63 \%$ within the first 72 hours of admission, of which $26 \%$ died [2]. Using the same criteria as the Santbrink study, in our study mortality was $88 \%$. There is significant mortality in patients in patients who initially have hypoperfusion and then subsequently normalize their measurements. Eight of 18 patients (44\%) with initial hypoperfusion that normalized, died in our study. Persistent hypoperfusion is almost invariably fatal.

Vasospasm occurred in $27 \%$ of our patients. The onset for vasospasm can occur from hospital day one to day 13, with days 2 and 3 being the most common [4]. Subarachnoid hemorrhage (SAH) can occur in as high as $60 \%$ of patients with TBI and is considered a negative prognostic risk factor. In our series $34 \%$ had a SAH as a significant component of their TBI, of these $40 \%$ died. Developing vasospasm following aneurysmal SAH is considered to be related to blood clot burden. Post-traumatic vasospasm can occur in the absence of SAH. Sixty-four percent of our patients with vasospasm did not have a SAH. This questions whether aneurysmal SAH and post-traumatic SAH share similar pathophysiology [9].

The treatment for vasospasm from a TBI remains a challenge. For aneurysmal SAH, calcium channel blockers reduce morbidity. A Cochrane Review meta-analysis suggested lack of efficacy for calcium channel blockers in the setting of TBI as a whole. When a subgroup analysis was performed, a small benefit in the patients with TBI and traumatic SAH was demonstrated [10].

We agree with Bouzat's review, TCD is useful in patients with severe TBI [11]. It is helpful in predicting outcome and management. For patients with normal measurements, over $80 \%$ can expect a good outcome and low mortality from brain death. Patients with hypoperfusion measurements have a high mortality. Patients with vasospasm measurements have an increased mortality of $32 \%$. They also have a less favorable outcome with $45 \%$ having a good outcome. The treatment of vasospasm is uncertain however a calcium channel blocker should be considered.

\section{References}

1. Ziegler D, Cravens G, Poche G, et al. Use of transcranial Doppler in patients with severe traumatic brain injury. J Neurotrauma. 2017;34(1):121-7.

2. Santbrink HV, Schouten JW, Steyerberg EW, et al. Serial transcranial Doppler measurements in traumatic brain injury with special focus on early posttraumatic period. Acta Neurochirugica. 2002;144:1141-9.

3. Ract C, Le Moigno S, Bruder N, et al. Transcranial Doppler ultrasound goal directed therapy for early management of severe traumatic brain injury. Intensive Care Med. 2007;33:645-51.

4. Oertel M, Boscardin WJ, Orbist WD, et al. Post-traumatic vasospasm: The epidemiology, severity and time course of an underestimated phenomenon: A prospective study performed in 299 patients. J Neurosurgery. 2005;103:812-24.

5. Aaslid R, Huber P, Normes H. Evaluation of cerebrovascular spasm with transcranial Doppler ultrasound. J Neurosurgery. 1894;60:37-41.

6. Lindegaard KF, Normes H, Bakke SI, et al. Cerebral vasospasm diagnosis by means of angiography and blood velocity measurements. Acta neurochirugica. 1989;100:12-24.

7. Zubkov AY, Lewis AI, Raila FA, et al. Risk factors for the development of post-traumatic cerebral vasospasm. Surg Neurol. 2000;53:126-30.

8. Jennett B, Bond M. Assessment of outcome after severe brain damage. Lancet. 1975;305:480-5.

9. Mattioli C, Beretta L, Gerevini S, et al. Traumatic subarachnoid hemorrhage on the computerized tomography obtained at admission: A multicenter assessment of the accuracy of diagnosis and the potential impact on patient outcome. J Neurosurg. 2003;98:37-42. 
10. Langham J, Goldrad C, Teasdale G, et al. Calcium channel blockers for acute traumatic brain injury, Cochrane Database Syst Rev. 2000;2:CD000565.
11. Bouzat P, Oddo M, Payen JF. Transcranial Doppler after traumatic brain injury: Is there a role? Curr Opin Crit Care. 2014;20:153-160.

\section{*Correspondence to:}

Ziegler Daniel, Department of Surgery, John Peter Smith Hospital, Fort Worth, Texas

USA

E-mail: dziegler@jpshealth.org 